OPEN ACCESS

Edited by:

András Székács,

National Agricultural Research and

Innovation Centre, Hungary

Reviewed by:

Antonio Figueira,

University of São Paulo, Brazil

Jeff Wolt,

lowa State University, United States

*Correspondence:

Pamela Bachman

pamela.bachman@climate.com

Specialty section:

This article was submitted to

Plant Microbe Interactions,

a section of the journal

Frontiers in Plant Science

Received: 29 October 2019

Accepted: 10 January 2020

Published: 06 February 2020

Citation:

Bachman P, Fischer J, Song Z, Urbanczyk-Wochniak E and Watson $G$

(2020) Environmental Fate and

Dissipation of Applied dsRNA in Soil,

Aquatic Systems, and Plants.

Front. Plant Sci. 11:21.

doi: $10.3389 /$ fpls.2020.00021

\section{Environmental Fate and Dissipation of Applied dsRNA in Soil, Aquatic Systems, and Plants}

\author{
Pamela Bachman ${ }^{1,2 *}$, Joshua Fischer ${ }^{2}$, Zihong Song ${ }^{2}$, Ewa Urbanczyk-Wochniak ${ }^{2}$ \\ and Greg Watson ${ }^{2}$ \\ 1 Science Organization, The Climate Corporation, Creve Coeur, MO, United States, ${ }^{2}$ Regulatory Science, Bayer Crop \\ Science, Chesterfield, MO, United States
}

Two primary use patterns exist for dsRNA-based products for crop protection: in planta produced dsRNA such as in a genetically engineered (GE) crop; and topically applied dsRNA such as a spray application. To enable effective environmental risk assessments for these products, dsRNA must be successfully measured in relevant environmental compartments (soil, sediment, surface water) to provide information on potential exposure. This perspective reviews results from numerous environmental fate and degradation studies with topically applied unformulated dsRNAs to demonstrate the high lability of these molecules and low potential for persistence in the environment. Additionally, we report on results of a pilot study of topically applied dsRNA on soybean plants demonstrating similar rapid degradation under field conditions. Microbial degradation of nucleic acids in environmental compartments has been shown to be a key driver for this lack of persistence. In fact, the instability of dsRNA in the environment has posed a challenge for the development of commercial topically-applied products. Formulations or other approaches that mitigate environmental degradation may lead to development of commercially successful products but may change the known degradation kinetics of dsRNAs. The formulation of these products and the resultant impacts on the stability of the dsRNA in environmental compartments will need to be addressed using problem formulation and product formulation testing may be required on a case by case basis to ensure an effective risk assessment.

Keywords: RNAi, dsRNA, environmental risk assessment, environmental fate, dissipation

\section{INTRODUCTION}

To conduct an effective environmental risk assessment (ERA) for a dsRNA-based, pesticidal agricultural product, it is necessary to determine the routes of exposure for non-target organisms (NTOs) and reliably quantify the concentration and persistence of the dsRNA in relevant environmental compartments such as plant tissues, soil, and surface waters/sediment. Two primary use patterns exist for dsRNA-based products in crop improvement: in planta produced dsRNA such as in a genetically engineered (GE) crop; and topically applied dsRNA such as a spray application. 
As discussed in Romeis and Widmer (2019), problem formulation is a core component of the ERA framework offering a logical approach and roadmap to characterize risk. Key to this approach is defining assessment endpoints, developing a conceptual model of predicted environmental relationships, and drafting an analysis plan to collect relevant data in regard to exposure and effects to perform a risk characterization (Nickson, 2008).

This perspective summarizes the current research on the environmental fate and degradation of dsRNA, with a focus on topically applied dsRNA, including exposure scenarios and quantification approaches, as well as identifying gaps in knowledge and key questions to be addressed in ERAs for dsRNA crop protection products.

\section{EXPOSURE SCENARIOS}

For in planta expressed dsRNA the concentration of dsRNA across tissues and growth stages can be used to estimate the maximum exposure levels to terrestrial and aquatic NTOs. Typically, samples are collected from multiple tissues (e.g. pollen, leaf, root) and analyzed across life stages of the plant to provide a thorough characterization of the expression of the dsRNA as NTOs may feed on or be exposed to specific plant tissues at specific life stages of the plant. The primary receiving compartment for in planta produced dsRNAs is the soil due to the incorporation of plant biomass post-harvest. Based upon a conceptual model of an in planta produced insecticidal dsRNA (Bachman et al., 2016), potential exposure to NTOs could occur through ingestion of the dsRNA containing tissues by herbivores and other soil dwelling organisms. Additionally, some plant material can move off-field into nearby surface waters and associated sediments as described in Carstens et al. (2012).

With topical application, it is possible to build upon the standard assumptions used for conventional pesticide sprays where soil is generally considered the primary receiving compartment in the environment with some off-site movement from spray (e.g. spray drift or soil run off) that may occur and could lead to NTO exposure in surface waters/sediments. For conventional pesticides, residue chemistry data are typically collected to provide the information necessary to determine the site, nature, and magnitude of residues in or on food/feed to estimate the exposure of the general population to pesticide residues and to set and enforce tolerances or maximum residue limits for pesticide residues in food/feed. For a topically applied dsRNA, the analysis of residues on plant tissues may provide additional data to inform the ERA as standard models for exposure of conventional sprayed pesticides (e.g. Kenaga nomogram) may overestimate the exposure of NTOs to sprayed dsRNA. For example, due to the barriers in plants to the uptake of sprayed dsRNA (e.g., cuticle, plant cell walls) the dsRNA applied to foliage would largely remain on the surface and be subject to environmental and microbially mediated degradation. As with conventional pesticides, the impact of product formulation such as stabilizing agents needs to be considered as part of the risk assessment, particularly if formulations are designed to overcome physical or biochemical barriers in target pests.

\section{QUANTIFICATION OF dsRNA}

The QuantiGene RNA assay has been used to accurately quantify dsRNA in environmental samples (Dubelman et al., 2014; Fischer et al., 2016; Albright et al., 2017; Fischer et al., 2017). This hybridization-based assay displays high specificity and can measure a single transcript from samples. It offers a highthroughput solution with repeatable results that have been accepted by regulatory agencies for product registration (U.S. EPA, 2017). Details on the use of QuantiGene can be found in Armstrong et al. (2013) with specifics on validation in soil matrices in Fischer et al. (2016). In side by side comparisons, QuantiGene results have been shown to be consistent with other methods for dsRNA detection such as northern blots, PCR, and UPLC (data not shown). The QuantiGene approach provides an advantage as it is more quantitative than a northern blot, less labor intensive, can quantify specific nucleic acid sequences unlike UPLC, and does not require amplification of the analyte as does PCR.

Parker et al. (2019) radiolabeled dsRNA with phosphorous-32 $\left({ }^{32} \mathrm{P}\right)$ and were able to quantify concentrations at the $\mathrm{ng} / \mathrm{g}$ soil level. This approach allowed for refinement over previous work with QuantiGene by assessing dsRNA adsorption to soil particles and bio-degradation as part of the overall degradation characterization. Labeled dsRNAs were shown to degrade rapidly in soil suspensions, adsorb to particle surfaces, and be utilized by soil microorganisms. However, radiolabeling as an analytical method for nucleic acids has limits, as the labeled nucleotides are scavenged by organisms as a nutrient source, potentially confounding the degradation assessment and estimates of total recoverable radioactivity (TRR) would be a conservative over estimate of residues. From an ERA perspective, as with conventional pesticides, the bioavailability of active ingredients bound to soil particles is a consideration since long segments of dsRNA are negatively charged biopolymers that have the ability to bind to soil particles (Greaves and Wilson, 1969; Trevors, 1996; Draper, 2004; Pietramellara et al., 2009). Relatively harsh extraction methods are normally employed for conventional chemicals to free active ingredients from soil particles, but this approach is not likely to be suitable for dsRNA as it could destroy the test material. However, as discussed below dsRNA bound to soil particles is not likely to be a significant contributor to the ERA given the demonstrated rapid degradation of dsRNA in soil and soil suspensions, and the need for dsRNA to be unbound (and therefore subject to rapid degradation) to have any biologically meaningful activity.

\section{FATE OF dsRNA IN SOIL, SURFACE WATERS, AND SEDIMENT}

Laboratory microcosm studies enable robust replication and sampling to quantitatively assess degradation rates of dsRNA that can be used in risk assessments. A comprehensive series of environmental fate and degradation studies were performed in soil, surface water, and sediment for the insecticidal DvSnf7 
dsRNA expressed in MON 87411 maize (Dubelman et al., 2014; Fischer et al., 2017). Results with DvSnf7 dsRNA are consistent with other published studies (Tabata et al., 1993; Zhu, 2006; Pietramellara et al., 2009; Eichmiller et al., 2016) that show nucleic acids are rapidly degraded in soil and aquatic environments. In these studies, a two-pronged approach was utilized employing both the QuantiGene assay and responsive insect bioassays to evaluate the environmental degradation of the dsRNA and the concurrent loss of functional bioactivity. This information was used to determine the potential exposure period for NTOs.

Dubelman et al. (2014) determined the biodegradation potential of the DvSnf7 dsRNA in three representative active agricultural soils with differing physicochemical characteristics. The estimated $\mathrm{DT}_{50}$ (time to $50 \%$ degradation) of the dsRNA in all soils was $<30$ hours and the $\mathrm{DT}_{90}$ (time to $90 \%$ degradation) values were $<35$ hours. These results combined with similar $\mathrm{DT}_{50}$ and $\mathrm{DT}_{90}$ values from insect bioassays demonstrating the loss of functional activity, indicate dsRNAs are unlikely to persist or accumulate in the soil, regardless of soil texture, $\mathrm{pH}$, clay content, or other differences. In addition, Dubelman et al. (2014) demonstrated that the degradation kinetics of DvSnf7 dsRNA are independent of the initial dsRNA concentration as soil samples spiked with dsRNA at 0.3-37.5 $\mu \mathrm{g} / \mathrm{g}$ soil displayed no apparent change in degradation kinetics.

Further work to elucidate the influence of dsRNA size, structure, and sequence on degradation kinetics was described in Fischer et al. (2016). The degradation of two dsRNA molecules with no significant shared sequence match and of different sequence lengths (968 and $100 \mathrm{bp}$ ) and structures (hairpin and linear) were evaluated in biologically active soil. The degradation kinetics of the two molecules were indistinguishable and displayed similar rapid degradation in soils as reported in Dubelman et al. (2014). These results suggest that unmodified dsRNAs are extremely labile and will not accumulate or persist in the environment. Joaquim et al. (2019) recently reported comparable results for DvSnf7 dsRNA degradation in tropical soils from Brazil.

DsRNAs have also been shown to degrade and not persist in aquatic systems, with half-lives of less than 3 days. Fischer et al. (2017) measured the degradation of DvSnf7 dsRNA in biologically active sediments and water collected from two separate natural systems representative of agricultural areas. The dsRNA was shown to rapidly degrade in the water phase of sediment-water microcosms. The dsRNA also degraded rapidly in a sediment-only system which lacked the overhead water column. As noted in Fischer et al. (2017), dsRNAs prepared in sterile (deionized) water appeared to be stable over the course of these studies, whereas the test systems utilized field collected and biologically active water and sediments indicating that the degradation of dsRNA is likely driven by microbial degradation. These results are consistent with previous work demonstrating that nucleic acids degrade rapidly and do not persist in aquatic compartments (Tabata et al., 1993; Zhu, 2006; Eichmiller et al., 2016).

To mimic the entry of a dsRNA into an aquatic system through either spray drift or transport by plant tissues,
Albright et al. (2017) examined the dissipation of dsRNA within the water column and potential partitioning into the sediment compartment. As seen in Fischer et al. (2017), dissipation in the water column was rapid [ $<$ limit of detection (LOD) after 96 hours]. Non-significant levels of dsRNA were observed in sediment which the authors conclude may be due to rapid degradation in the water column precluding portioning into the sediment.

\section{FATE OF dsRNA IN FOLIAR APPLICATIONS}

There is a paucity of data describing the fate of foliarly-applied dsRNAs, and the data that are available is contradictory. Differences have been observed in the post application stability of the sprayed dsRNA product within controlled environments versus preliminary data from field environments and different detection/quantification methods have been employed that make comparison across studies difficult.

Mitter et al. (2017a; 2017b) reported that dsRNA suspensions sprayed on leaf surfaces under controlled conditions only offered 5 days of virus protection before degrading as confirmed by northern blot. Additionally, Cy3-labeled dsRNA applied to leaf surfaces and rinsed after 24 hours to mimic a rain event demonstrated that the dsRNA readily washed away as determined by confocal microscopy (Mitter et al., 2017a).

In a greenhouse experiment, San Miguel and Scott (2016) observed efficacy of up to 28 days for an insecticidal dsRNA applied to potato leaves. The dsRNA was not readily washed off once it had dried on the potato leaves. When the same dsRNA was incorporated into a gel and exposed to UV light for 1-2 hours, it was shown to be inactive. No quantification of the dsRNA used in these experiments was performed, but a responsive insect bioassay was used to determine the presence of active dsRNA for these studies.

In 2014 Bayer Crop Science conducted a pilot study to determine the magnitude and decline of residue levels of a topically applied $100 \mathrm{bp}$ dsRNA on soybean under field conditions. The dsRNA was the same $100 \mathrm{bp}$ sequence as used in Fischer et al. (2016) and displayed rapid dissipation in soil. The study was conducted under procedures consistent with Good Laboratory Practices (GLP).

The study site was in Puerto Rico and the soybean was produced under agronomic conditions and practices typical in that region. The study consisted of a single untreated control plot (treatment 1) and two treatment plots (treatments 2 and 3) with two replicates each (Table 1). In treatment 2, dsRNA was applied at target rate of $59.3 \mathrm{~g}$ ai/ha at three separate applications: V4/R1, V10/R3, and 7 days before harvest whereas treatment 3 omitted the 7 day preharvest treatment. Each plot consisted of four rows planted on $0.76 \mathrm{~m}$ rows that were $15.2 \mathrm{~m}$ long (approximately $46.5 \mathrm{~m}^{2}$ plot area). The seed used was a commercial variety of RoundUp Ready/Insect Protected soybean (Asgrow). Applications were made with a backpack $\mathrm{CO}_{2}$ sprayer with a flat fan nozzle. Weather during the study was similar to the historical average (mean temperature $22.1-30.7^{\circ} \mathrm{C} ; 9.5 \mathrm{~cm}$ mean 
rainfall) and no rain events were recorded during the spraying or whole plant residue collection period. Irrigation was provided via drip tape. Aerial portions (above soil) of the plants were collected to determine residue levels of the dsRNA via the Quantigene assay. Whole plant samples were immediately frozen on dry ice and maintained frozen on dry ice or at $-80^{\circ} \mathrm{C}$ until analysis. No growth or developmental abnormalities were observed during the field study.

Contrary to results reported from similar experiments in controlled environments, under field conditions the concentration of the foliarly-applied dsRNA rapidly declined with a $\sim 95 \%$ reduction 3 days after treatment (DAT) and an almost $99 \%$ reduction 7 DAT. The estimated dissipation kinetics provide a $\mathrm{DT}_{50}$ of 0.7 days and a $\mathrm{DT}_{90}$ of 2.3 days for treatment 2 and $\mathrm{DT}_{50}$ of 0.5 days and $\mathrm{DT}_{90}$ of 1.9 days for treatment 3 (Figure 1). Additionally, negligible amounts $(0.19 \mathrm{ng} / \mathrm{g} \mathrm{fw})$ of dsRNA were detectable in harvested grain from the soy plants at maturity in treatment 2 , which included an application of

TABLE 1 | Summary of pilot field study to evaluate stability of topically applied dsRNA on soybean plants using the QuantiGene assay.

\begin{tabular}{|c|c|c|c|c|c|}
\hline Treatment & $\begin{array}{l}\text { Application } \\
\text { rates } \\
\text { (target) and } \\
\text { timing }\end{array}$ & $\begin{array}{l}\text { Spray } \\
\text { rate } \\
\text { (liters } \\
\text { per } \\
\text { hectare) }\end{array}$ & Carrier & Sampling & $\begin{array}{l}\text { Residue level } \\
\text { average } \\
\text { concentration }{ }^{4} \\
\text { ng/g fw (min.- } \\
\text { max.) }\end{array}$ \\
\hline $\begin{array}{l}1 \text { (Control, } \\
1 \text { plot) }\end{array}$ & Untreated & 187 & $\begin{array}{l}\text { Ultrapure } \\
\text { water } \\
\text { plus } \\
\text { Silwet L- } \\
77 \text { at }\end{array}$ & $\begin{array}{l}12 \text { whole } \\
\text { plants; } \\
\text { plus } 1 \mathrm{~kg} \\
\text { grain at } \\
\text { maturity }\end{array}$ & $\mathrm{N} / \mathrm{A}$ \\
\hline $\begin{array}{l}2(2 \\
\text { replicate } \\
\text { plots) }\end{array}$ & $\begin{array}{l}59.3 \mathrm{~g} \text { ai/ha } \\
\text { at three } \\
\text { separate } \\
\text { applications: } \\
\text { V4/R1, V10/ } \\
\text { R3, and } 7 \\
\text { days before } \\
\text { harvest }\end{array}$ & 187 & $0.5 \% \mathrm{v} / \mathrm{v}$ & $\begin{array}{l}12 \text { whole } \\
\text { plants per } \\
\text { plot } \\
\text { collected } \\
\text { and } \\
\text { pooled for } \\
\text { analysis at } \\
0,3 \text {, and } 7 \\
\text { DAT² ; plus } \\
1 \text { kg grain } \\
\text { at maturity }\end{array}$ & $\begin{array}{c}\text { O DAT }{ }^{2}: 4166 \\
(4158-4174) \\
\text { 3 DAT }: 242 \\
(235-249) \\
\text { 7 } \text { DAT }^{2}: 66.23 \\
(56.01-76.44) \\
\text { Seeds: } 0.19 \\
(0.15-0.23)\end{array}$ \\
\hline $\begin{array}{l}3(2 \\
\text { replicate } \\
\text { plots) }\end{array}$ & $\begin{array}{l}59.3 \mathrm{~g} \text { ai/ha } \\
\text { at two } \\
\text { separate } \\
\text { applications: } \\
\text { V4/R1, and } \\
\text { V10/R3 }\end{array}$ & 187 & & $\begin{array}{l}12 \text { whole } \\
\text { plants per } \\
\text { plot } \\
\text { collected } \\
\text { and } \\
\text { pooled for } \\
\text { analysis at } \\
0,3 \text {, and } 7 \\
\text { DAT }^{3} \text {; plus } \\
1 \text { kg grain } \\
\text { at maturity }\end{array}$ & $\begin{array}{c}\text { O DAT }^{3}: 1317 \\
(1014-1619) \\
\text { 3 DAT }: 50.33 \\
(50.17-50.49) \\
7 \text { DAT }^{3}: 25.66 \\
(25.47-25.85) \\
\text { Grain: Not } \\
\text { Detected (< } \\
\text { LOD) }\end{array}$ \\
\hline
\end{tabular}

${ }^{1}$ For treatment 2 actual applied rates were 54.9-63.9 g ai/ha. For treatment 3 actual applied rates were 57.2-59.4 $\mathrm{g}$ ai/ha.

${ }^{2}$ Days after treatment (DAT) refers to time point following initial application at V4/R1.

${ }^{3}$ Days after treatment (DAT) refers to time point following second application at V10/R3.

${ }^{4}$ The average residue concentration was calculated at each sampling interval for two replicate plots for treatments 2 and 3. dsRNA concentrations for each replicate plot provided in parenthesis.

Assay's LOD, $0.0015 \mathrm{ng} / \mathrm{g}$ fw; Assay's LOQ, $0.0180 \mathrm{ng} / \mathrm{g} \mathrm{fw;} \mathrm{fw,} \mathrm{fresh} \mathrm{weight;} \mathrm{N/A,} \mathrm{not}$ applicable.
dsRNA 7 days prior to grain harvest. No detectable residues of the dsRNA in grain was found in treatment 3 which lacked the 7 day preharvest treatment, thus supporting the conclusion that dissipation of the foliarly applied dsRNA was due to decline of residues on or near the plant surface and not due to uptake and degradation within the plant vascular system. This conclusion is supported by a similar GLP field study (not reported) performed with potatoes in three locations in the United States (Iowa, Wisconsin, and Washington) where two applications of dsRNA at $59.3 \mathrm{~g}$ ai/ha (4-5 weeks after planting and 28 days after the initial application) of the same $100 \mathrm{bp}$ dsRNA and a 397 bp dsRNA with activity against Colorado Potato Beetle did not result in detectable residues in potato tubers.

Several reasons may exist for the observed instability post application including photodegradation, wash-off due to rain or dew, and microbial degradation. UV light is known to degrade nucleic acids (Kundu et al., 2004) and San Miguel and Scott (2016) observed that dsRNA lost biological activity after exposure to UV light. Contrasting results were shown by San Miguel and Scott (2016) and Mitter et al. (2017a; 2017b) in terms of stability of sprayed dsRNA after washing, however no rainfall was recorded during the 7 DAT in the Bayer study. The rapid degradation of topically applied dsRNA in field versus controlled environments is not unexpected given the lability of nucleic acids in the environment and rapid degradation in the presence of microbes (Pietramellara et al., 2009; Parker et al., 2019).

Strategies to mitigate degradation could come from the formulation of end products such as addition of UV protectants, rain-fastness agents, and/or antimicrobials or physical encapsulations to limit microbial activity. In an environmental study in which dsRNA was protected by formulation ingredients by incorporation of dsRNA into

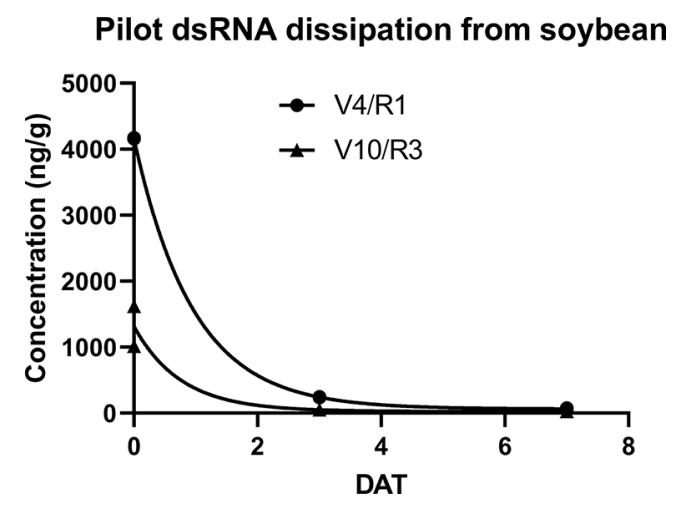

FIGURE 1 | dsRNA dissipation in soybean for treatments 2 (labeled V4/R1) and treatment 3 (labeled V10/R3) from pilot study. Treatment 2 measurements occurred at 0, 3, and 7 DATs of initial application at V4/R1. Treatment 3 measurements occurred at 0,3, and 7 DATs of second application at V10/R3. Estimated dissipation rate kinetics for V4/R1 are: $\mathrm{DT}_{50}$ of 0.7 days and $\mathrm{DT}_{90}$ of 2.3 days. Estimated dissipation rate kinetics for $\mathrm{V} 10$ / R3 are: $\mathrm{DT}_{50}$ of 0.5 days and $\mathrm{DT}_{90}$ of 1.9 days. Dissipation curves and estimates calculated from plotted individual replicates using a first order exponential decay model in Prism GraphPad v8.2.0. No error bars are illustrated as individual replicates are shown. 
layered double hydroxide ( $\mathrm{LDH})$ nanosheets known as "BioClay", virus protection of dsRNA applied to tobacco leaf surfaces was increased and extended from 5 to 20 days (Mitter et al., 2017a). Whitfield et al. (2018) demonstrated that cationic polymers applied to soil affect degradation kinetics and increase the lifetime of dsRNA in soil. Persistence of dsRNA in soil of up to 3 weeks was achieved through the application of a shaped poly(2-(dimethylamino) ethyl acrylate) analog. Given that these studies were done in protected environments, information is not yet available as to how these formulations will directly or indirectly impact NTOs or exposure scenarios for the dsRNAs contained in them.

\section{DISCUSSION}

To enable effective ERAs for dsRNA crop protection products, the dsRNA must be successfully measured in relevant environmental compartments based on intended use patterns. For topically applied dsRNA products, the primary environmental compartments are treated plants, soil, and secondarily surface waters/sediment. The QuantiGene assay is an appropriate and efficient analytical method for determining the environmental fate of dsRNA agricultural products and has been used successfully in registration applications for transgenic plants expressing insecticidal dsRNAs. Additional methodologies such as radiolabeling dsRNA offer potential refinements to the exposure assessment and may be useful to answer questions regarding the binding of dsRNA to soil particles versus degradation due to the potential confounding use of labeled nucleotides as a nutrient source. This technique should only be used as part of problem formulation for a given product or use pattern if further refinement of the exposure scenario is required. Standardization of analytical methods for quantification of dsRNA in environmental matrices will enhance the reconstructability, repeatability, and comparison of these types of studies and provide benefits to the regulatory process for dsRNA product approval.

Results from numerous environmental fate studies with unformulated dsRNAs demonstrate a high lability of these molecules and low potential for persistence in the environment including soil, sediment, and surface water compartments. Microbial degradation of nucleic acids in environmental compartments has been shown to be a key driver for this rapid

\section{REFERENCES}

Albright, V. C.III, Wong, C. R., Hellmich, R. L., and Coats, J. R. (2017). Dissipation of double-stranded RNA in aquatic microcosms. Environ. Toxicol. Chem. 36, 1249-1253. doi: 10.1002/etc.3648

Armstrong, T. A., Chen, H., Ziegler, T. E., Iyadurai, K. R., Gao, A. G., Wang, Y., et al. (2013). Quantification of transgene-derived double-stranded RNA in plants using the QuantiGene nucleic acid detection platform. J. Agr. Food Chem. 61, 12557-12564. doi: 10.1021/jf4031458

Bachman, P. M., Huizinga, K. M., Jensen, P. D., Mueller, G., Tan, J., Uffman, J. P., et al. (2016). Ecological risk assessment for DvSnf7 RNA: a plant-incorporated protectant with targeted activity against western corn rootworm. Regul. Toxicol. Pharm. 81, 77-88. doi: 10.1016/j.yrtph.2016.08.001 degradation and lack of persistence. Preliminary results suggest that foliarly-applied dsRNA is subject to rapid degradation under field conditions. For these dsRNA products, more data are needed to understand the drivers of stability on leaf surfaces especially under field conditions as low environmental stability could affect product efficacy. Modifications to dsRNA or formulations that alter stability in the environment, or overcome physical or biochemical barriers in target pests, may require additional studies to determine their effects on dissipation and degradation rates and any potential increase in exposure to relevant NTOs.

Formulations or other approaches to mitigate environmental degradation may lead to more successful products but may change the known degradations kinetics of dsRNAs. The formulation of these products and the resultant impacts on the stability of the dsRNA in environmental compartments will need to be addressed in problem formulation on a case by case basis to ensure an efficient risk assessment.

\section{DATA AVAILABILITY STATEMENT}

The datasets generated for this study are available on request to the corresponding author.

\section{AUTHOR CONTRIBUTIONS}

$\mathrm{PB}, \mathrm{JF}$, and GW conceptualized the content of the manuscript. $\mathrm{PB}$ wrote the first draft of the manuscript. EU-W and GW designed the pilot studies. ZS developed and validated the residue analytical method and conducted the residue analysis. All authors contributed to the manuscript revision, read, and approved the submitted version.

\section{ACKNOWLEDGMENTS}

The authors wish to thank Sonya Franklin, Kara Giddings, Kristin Huizinga, Steve Levine, and John Vicini of Bayer Crop Science for their constructive feedback during the preparation of this manuscript.

Carstens, K., Anderson, J., Bachman, P., De Schrijver, A., Dively, G., Federici, B., et al. (2012). Genetically modified crops and aquatic ecosystems: considerations for environmental risk assessment and non-target organism testing. Transgenic Res. 21, 813-842. doi: 10.1007/s11248-011-9569-8

Draper, D. E. (2004). A guide to ions and RNA structure. RNA 10, 335-343. doi: $10.1261 /$ rna.5205404

Dubelman, S., Fischer, J., Zapata, F., Huizinga, K., Jiang, C., Uffman, J., et al. (2014). Environmental fate of double-stranded RNA in agricultural soils. PloS One 9, e93155. doi: 10.1371/journal.pone.0093155

Eichmiller, J. J., Best, S. E., and Sorensen, P. W. (2016). Effects of temperature and trophic state on degradation of environmental DNA in lake water. Environ. Sci. Technol. 50, 1859-1867. doi: 10.1021/acs.est.5b05672 
Fischer, J. R., Zapata, F., Dubelman, S., Mueller, G. M., Jensen, P. D., and Levine, S. L. (2016). Characterizing a novel and sensitive method to measure dsRNA in soil. Chemosphere 161, 319-324. doi: 10.1016/j.chemosphere.2016.07.014

Fischer, J. R., Zapata, F., Dubelman, S., Mueller, G. M., Uffman, J. P., Jiang, C., et al. (2017). Aquatic fate of a double-stranded RNA in a sediment-water system following an overwater application. Environ. Toxicol. Chem. 36, 727-734. doi: 10.1002/etc.3585

Greaves, M. P., and Wilson, M. J. (1969). The adsorption of nucleic acids by montmorillonite. Soil Biol. Biochem. 1, 317-323. doi: 10.1016/0038-0717(69)90014-5

Joaquim, M. E. S., Belchior, G. G., José, M. O. D. M. A., Zapata, F., Jiang, C., Fischer, J., et al. (2019). Dissipation of DvSnf7 Double-Stranded RNA in Brazilian Soils. Agr. Environ. Let. 4, 1-4. doi: 10.2134/ael2019.04.0016

Kundu, L. M., Linne, U., Marahiel, M., and Carell, T. (2004). RNA is more UV resistant than DNA: the formation of UV-induced DNA lesions is strongly sequence and conformation dependent. Chemistry-A Eur. J. 10, 5697-5705. doi: 10.1002/chem.200305731

Mitter, N., Worrall, E. A., Robinson, K. E., Li, P., Jain, R. G., Taochy, C., et al. (2017a). Clay nanosheets for topical delivery of RNAi for sustained protection against plant viruses. Nat. Plants 3, 16207. doi: 10.1038/nplants.2016.207

Mitter, N., Worrall, E. A., Robinson, K. E., Xu, Z. P., and Carroll, B. J. (2017b). Induction of virus resistance by exogenous application of double-stranded RNA. Curr. Opin. Virol. 26, 49-55. doi: 10.1016/j.coviro.2017.07.009

Nickson, T. E. (2008). Planning environmental risk assessment for genetically modified crops: problem formulation for stress-tolerant crops. Plant Physiol. 147, 494-502. doi: 10.1104/pp.108.118422

Parker, K. M., Barragán Borrero, V., Van Leeuwen, D. M., Lever, M. A., Mateescu, B., and Sander, M. (2019). Environmental fate of RNA interference pesticides: adsorption and degradation of double-stranded RNA molecules in agricultural soils. Environ. Sci. Technol. 53, 3027-3036. doi: 10.1021/acs.est.8b05576

Pietramellara, G., Ascher, J., Borgogni, F., Ceccherini, M. T., Guerri, G., and Nannipieri, P. (2009). Extracellular DNA in soil and sediment: fate and ecological relevance. Biol. Fert. Soils 45, 219-235. doi: 10.1007/s00374-008-0345-8

Romeis, J., and Widmer, F. (2019). Assessing the risks of topically applied dsRNAbased products to non-target arthropods. Front. Plant Sci.
San Miguel, K., and Scott, J. G. (2016). The next generation of insecticides: dsRNA is stable as a foliar-applied insecticide. Pest Manage. Sci. 72, 801-809. doi: 10.1002/ps.4056

Tabata, M., Takada, Y., Sato, M., Suzuki, J., and Suzuki, S. (1993). Distributions of DNA and RNA hydrolyzing bacteria in lakes and their extracellular nuclease production. Jpn. J. Limnol. 54, 117-123. doi: 10.3739/rikusui.54.117

Trevors, J. T. (1996). DNA in soil: adsorption, genetic transformation, molecular evolution and genetic microchip. Anton. Leeuw. 70, 1-10. doi: 10.1007/ BF00393564

U.S. EPA (2017). Registration decision for commercial use corn products containing the DvSnf7 dsRNA plant-incorporated protectant (Event MON 87411). Available from https://www.regulations.gov/document?D=EPA-HQOPP-2014-0293-0407.

Whitfield, R., Anastasaki, A., Truong, N. P., Cook, A. B., Omedes-Pujol, M., Loczenski Rose, V., et al. (2018). Efficient binding, protection, and self-release of dsrna in soil by linear and star cationic polymers. ACS Macro Lett. 7, 909915. doi: 10.1021/acsmacrolett.8b00420

Zhu, B. (2006). Degradation of plasmid and plant DNA in water microcosms monitored by natural transformation and real-time polymerase chain reaction (PCR). Water Res. 40, 3231-3238. doi: 10.1016/j.watres.2006.06.040

Conflict of Interest: The research reported was funded by Bayer Crop Science and the researchers involved in this work were employees of Bayer Crop Science and its predecessors in business.

Copyright (C) 2020 Bachman, Fischer, Song, Urbanczyk-Wochniak and Watson. This is an open-access article distributed under the terms of the Creative Commons Attribution License (CC BY). The use, distribution or reproduction in other forums is permitted, provided the original author(s) and the copyright owner(s) are credited and that the original publication in this journal is cited, in accordance with accepted academic practice. No use, distribution or reproduction is permitted which does not comply with these terms. 